\title{
FREE-FORM TOOLS DESIGN AND FABRICATION FOR FLANK SUPER ABRASIVE MACHINING (FSAM) NON DEVELOPABLE SURFACES
}

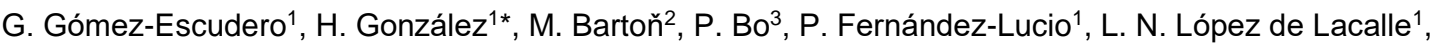 \\ A. Calleja ${ }^{1}$ \\ ${ }^{1}$ University of the Basque Country, Torres Quevedo s/n, 48013 Bilbao, Vizcaya, Spain \\ ${ }^{2}$ BCAM - Basque Center for Applied Mathematics, Alameda de Mazarredo 14, 48009 Bilbao, Vizcaya, Spain \\ ${ }^{3}$ School of Computer Science and Technology, Harbin Institute of Technology, West Wenhua Street 2, 264209 \\ Weihai, China \\ *Corresponding author; e-mail: haizea.gonzalez@ehu.eus
}

\begin{abstract}
Manufacturing improvements are becoming a real need in industry. In order to satisfy these industrial requirements, they should be targeted in two different directions: new manufacturing processes and surface optimization through algorithms. On the one hand, Super Abrasive Machining (SAM) is presented as a new manufacturing process combining benefits from milling and grinding technologies. On the other hand, there is a tendency to manufacture non developable surfaces by flank milling and to achieve final dimensional and roughness requirements, by calculating mathematically-optimized tool trajectories.

This work presents a design and manufacturing of a free form tool to be used for the manufacturing of a complex surface through Flank SAM (FSAM). Based on the tool requirements, it will cover the following stages: tool geometry design, tool core manufacturing, and electroplating for final abrasive tool generation.
\end{abstract}

\section{Keywords:}

Free form abrasive tool; Non developable surfaces; Flank Super Abrasive Machining (FSAM)

\section{INTRODUCTION}

The aeronautical sector continues the current trend of applying stricter standards on efficiency, noise, and fuel consumption. Consequently, airplane manufacturing companies will have to optimize their manufacturing processes in order to be competitive [Chaves-Jacob 2012]. It should be paid special attention to turbomachinery rotary components such as impellers or blisks, because of the influence on the final cost of the aircraft engine. Moreover, for these components, the performance is particularly dependent on manufacturing accuracy, and that is the main reason why many researches have been carried out in the last years trying to optimize manufacturing processes of these components [Klocke 2015], [Wang 2018].

These engine components play a fundamental role in the stages of both compression and expansion of gas inside the aircraft turbines. The components transfer energy between a gas flow and a mechanical part; this function is performed by the geometric evolution of the flow passage. This passage is delimited by two adjacent blades: intrados and extrados. Thus, these two surfaces are constrained by functional requirements expressed by mechanical, hydraulic and machining laws. Therefore, their surface finishing operation is fundamental. At present, two methods of finishing are widely spread in aeronautic industry: point milling and flank milling. Point milling uses the end of the tool to remove the material, whereas flank milling uses the flank of the tool. Point milling suits every type of geometry, but its material removal rate is smaller in comparison with flank milling. In addition, compared with point milling, flank milling presents a longer tool life and higher surface quality. However, it is very difficult to control finishing machining errors for using this flank milling strategy on complex freeform surfaces manufacturing processes due to the complexity of calculating the tool path. Therefore, many researchers focus on how to find precise and robust methods to calculate the tool path for flank milling in the last decades, e.g. [Menzel 2004], [Zhu 2010], [Yi 2018] and [Li 2019].

Turbomachinery rotary components are designed by free form surfaces [Chuang 2007] which implies a difficulty to machine these components. In the last years, due to the tightening of specifications in regards to surface finish quality, the geometries have become more complicated, being transformed into non developable wrapped surfaces. This presents a complication in the programming of machining trajectories. In this sense, several studies focus on the optimization of tool positioning trajectories and tool geometry in the machining of turbomachinery rotary components. [Gong 2005] Focus on the optimization of the positioning (for each tool-tip point) of cylindrical tool with the aim of approximating non-developable surfaces to ruled surfaces, which is a particular geometrical type of surface obtained by the motion of a ruled surface along a defined guide. For developable surfaces (i.e., surfaces with constant normals along rulings), flank CNC machining is 
trivial as the grazing curve is the contact ruling. The real challenge of flank machining lies in general free form geometries. Then, the following algorithms were developed for conical tools, so flank milling operation can be performed on ruled surfaces [Lu 2016]. Nowadays, developed algorithms calculate both the optimal geometry of the tool and the machining trajectories, therefore, flank milling operation can be performed in non-ruled surfaces. [Bo 2016] developed an algorithm that computes the optimal shape of a general rotational tool and its motion in 3D space, such that the corresponding envelope approximates the input free form surface within a high accuracy.

Another factor that should be taken into account in the manufacturing of turbomachinery integral rotary components (IBRs), consists on the manufacturing process optimization in terms of time and cost. For blisk production, the manufacturing process starts commonly from a solid disk, thus a lot of material needs to be removed. Especially the milling process of blisks made of $\mathrm{Ni}$-based alloys has nearly reached its technological and economical limit. Therefore, it seems to be reasonable to use unconventional manufacturing technologies in the manufacturing process. Thereby, Flank Super Abrasive Machining (FSAM) is presented as a solution because it combines the benefits of the use of grinding tools with milling feed rates and flank milling strategies. SAM process has some advantages over single point machining process (Petrilli 2012): the design of the tools is simpler since there are no flutes; moreover, the structure of the tool is more robust; the maintenance that must be given to the tool is less and ,with this type of tools, post machining distortion is eliminated.

The tools that are used in SAM machining operations are mono-layer electroplated tools with superabrasive grains.. In this way, the use of this type of tools entails a saving in the cost of tools. Since they appeared until a few years ago, they have been generally used in turning/grinding operations in a wide range of applications in the automotive, optical [Stephenson 2006], and communication industry as well as in medical and life science in order to obtain ultraprecision components. Nowadays, some researchers study the use of electroplated milling tools in drilling [Biermann 2017] and milling [González 2018]. An electroplated milling tool is characterized by a steel core and a single or multi-layer of abrasive grains in the cutting area. During machining operations, abrasive grains are removed significantly rapid, so tool life will be short in comparison with milling tools. Hence, to improve the tool life, the grain bonding strength of the coatings, which bury diamond grains onto the shank, must be improved. The grain bonding strength is primarily determined by the following factors [Lin 1998], [De Resende 2001]: (1) adherence strength between grains and bond matrices; (2) mechanical properties of bond matrices (such as yield strength and stiffness) [Tanaka 1997]; (3) anchor effects related to the embedded depth of the grains by a bond matrix; (4) wear resistance of the bond matrix that is scratched by chips and worn out in the grinding process; and lastly; (5) friction coefficient of the bond matrix preventing material adhesion (wear type known as heavy loadings). The grain bonding strength can be enhanced by employing functional fillers in the bone matrix, thereby improving the above mentioned factors [Tsai 2012]. Nevertheless, when the diameter of the grain decreases, the thickness of the bond layer will be smaller, and the filler size is also smaller.

Combining previously defined directions, SAM tool with flank milling operation strategies, this work presents the design and manufacturing of a free form tool to be used for the manufacturing of a complex surface through Flank SAM. Based on the tool requirements, it will cover the following stages: tool geometry design, tool core manufacturing and electroplated for final abrasive tool generation.

\section{METHOD AND MATERIAL}

This work presents the design and manufacturing process of a free-form tool according to the geometry of a blisk blade. It is divided into three sections; firstly, the design of the tool according to a mathematic algorithm; then, the manufacturing of the core of the tool; and finally, the coating of the cutting side of the tool with abrasive grains.

\subsection{Design of the tool}

Until now, most of the mathematical algorithms proposed are focused on the approximation of complex surfaces by using small patches creating a whole envelope offering improved characteristics, i.e. ruled surfaces. In addition, along with the above, the flank milling method is used, because the entire cutting edge is used when machining, so machining time is decreased considerably.

What has been said above, brings two challenges to calculate the free-form geometry machining trajectories, the first one was consists of approximating large patches with a single sweep of one tool accomplishing allowed error values, and the second one consists on finding the optimal tool shape in order to offer the best approximation for flank milling to a given free form geometry.

A mathematic algorithm developed by Bo et al. [Bo 2016] was used in order to obtain the best fitting design of the tool. This algorithm considers the shape of the cutting side as a variable, so given the geometry to manufacture as an entrance parameter, that in this case is the convex side of the blade shown in Fig.1, it calculates the best geometry of the tool within a machining error of approximation to the surface. The designed tool of revolution is created considering the blade surface generatrix and the admissible final tolerances. Fig. 3 shows more in detail the tool section where it is appreciated the variability of the tool cutting area.

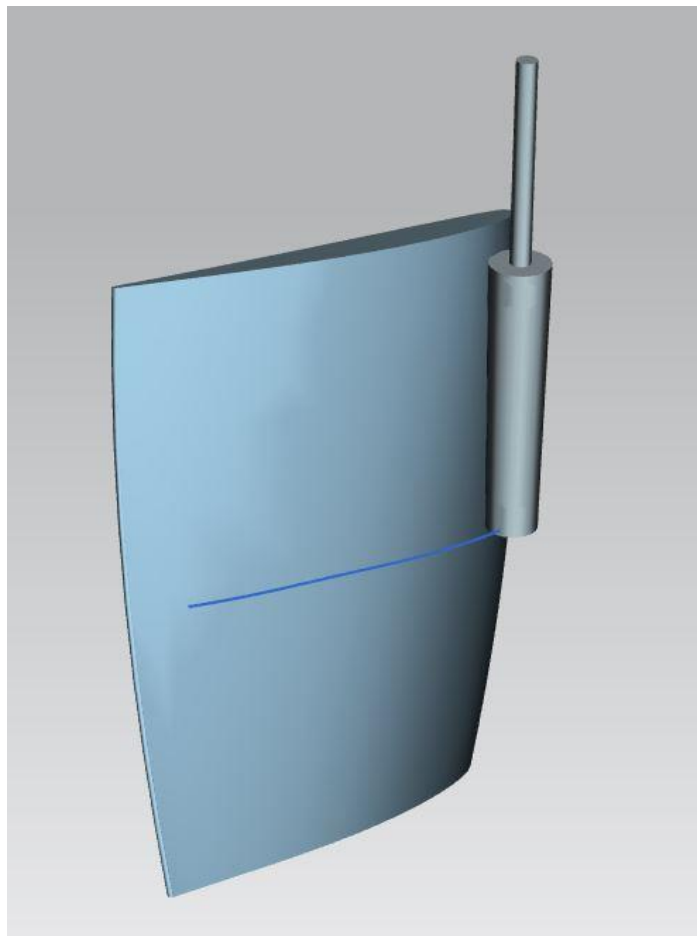

Fig. 1: Free-form revolution tool adapted to convex surface. 


\subsection{Tool core manufacturing}

\section{Material}

The selected material for the manufacturing of the tool core is AISI 1055 steel. Abrasive grains are easily electroplated to AISI 1055 steel. The chemical composition of this steel is shown in Tab. 1, and the mechanical and thermal properties are shown in Tab. 2.

Tab. 1: Chemical composition of AISI 1055 steel taken from standard.

\begin{tabular}{ccccc}
\hline $\mathbf{C}$ & $\mathbf{F e}$ & $\mathbf{M n}$ & $\mathbf{P}$ & $\mathbf{S}$ \\
\hline $0.55 \%$ & $98.65 \%$ & $0.75 \%$ & $0.02 \%$ & $0.03 \%$ \\
\hline
\end{tabular}

Tab. 2: Mechanical and thermal properties of steel AISI 1055 taken from standard.

\begin{tabular}{cc}
\hline Property & Value \\
\hline Density $\left(\mathrm{g} / \mathrm{cm}^{3}\right)$ & 7.85 \\
Thermal conductivity $(\mathrm{W} / \mathrm{m} \mathrm{K})$ & 48.9 \\
Specific heat $(\mathrm{J} / \mathrm{g} \mathrm{K})$ & 0.472 \\
Vickers hardness & 207 \\
Melting point $\left({ }^{\circ} \mathrm{C}\right)$ & 1460 \\
Elasticity modulus $(\mathrm{GPa})$ & $190-210$ \\
Tensile strength, yield $(\mathrm{MPa})$ & 560 \\
Tensile strength, ultimate $(\mathrm{MPa})$ & 660 \\
\hline
\end{tabular}

\section{Machine}

The manufacturing of the tool core was carried out on AIS 1055 steel in CNC turning center (CMZ TC25 BTY with FANUC 31iT numerical control), see Fig. 2. The lathe is equipped with four axes, three of them linear $X, Y$ and $Z$ and one rotary $\mathrm{C}$.

The clamping of the initial billet was done with a three-jaw chuck and without a counterpoint due to the small diameter of the tool.

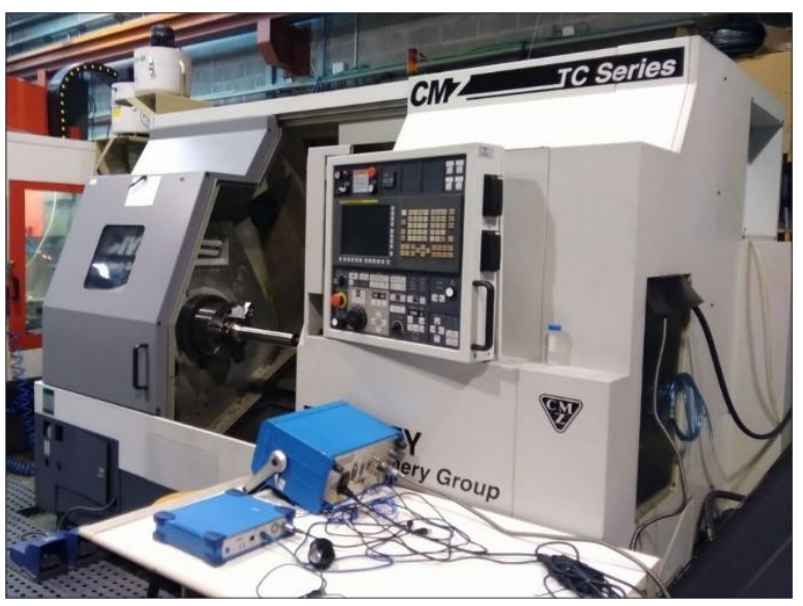

Fig. 2: CMZ TC25 BTY Turning center.

\section{Procedure}

To manufacture the core of the tool, a cylindrical billet of $40 \mathrm{~mm}$ of diameter of AISI 1055 steel was used. For this purpose, three different types of operations were defined. The first operation was a roughing operation in order to eliminate the most amount of material, and then a semifinishing operation was performed to approximate to the final size- In this last operation, cutting conditions were more conservative to reduce surface irregularities and improve the surface for the final finishing operation to get the designed geometry of the tool. Cutting conditions are shown in Tab.3.

The programming of the roughing operation was done directly programmed in the turning machine with a NC code, because removed material did not affect the final geometry of the tool itself. Whereas the trajectories of both semifinishing and finishing were programmed by a more complex CAM environment (due to the variability of the tool section), using the software Siemens NX12, shown in Fig. 3. This procedure was required due to the fact that, even though the tool surface is near to a cylindrical tool, the cutting zone is generated as a junction of different patches, obtaining a free-form surface. Therefore, this implies the no possibility to be programmed without a CAM environment that recognises exactly (with minimal error) the surface to be manufactured.

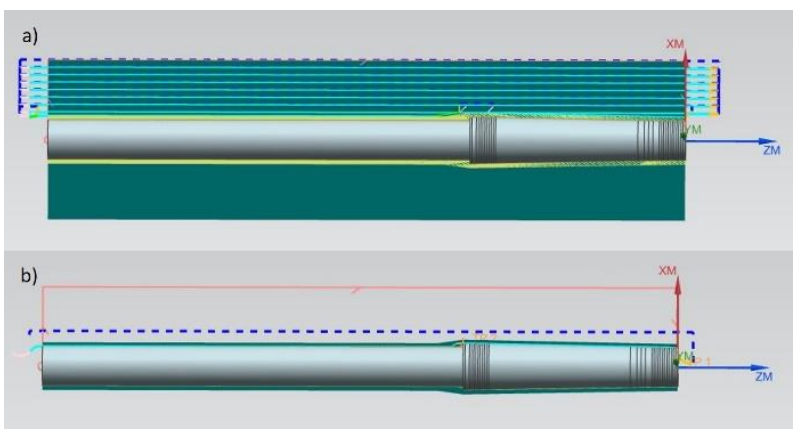

Fig. 3: Tool geometry manufacturing machining trajectories programmed in CAM. a) Top part: Semifinishing operation trajectories and b) Bottom part: Finishing operation trajectory.

The main objective for the roughing operation consists on removing the most amount of material in a quick manner. For this purpose, a square roughing insert was used with the denomination CNMG 1204 12-PR 4225 (Sandvik Coromant $($ ). With this insert five roughing turning passes were made with a depth of cut of $2 \mathrm{~mm}$, decreasing the initial diameter of the billet from 40 to 20 millimetres.

The semi-finishing operation is performed to achieve a surface near to the final size offering better surface quality than roughing. For this purpose, smaller tool diameter was used, around $6 \mathrm{~mm}$, so it was used a finishing rhombic insert denominated VBMT 1604 08-PR 4225 (Sandvik Coromant $\circledast$ ). After this operation performance, it was programmed a stock of $0.5 \mathrm{~mm}$ for the finishing operation.

Finally, a finishing pass was made to obtain the final geometry of the tool core, see Fig. 5 . In this operation the rhombic insert was also used, but applying a smaller depth of cut for optimal finishing quality, in this case $0.5 \mathrm{~mm}$. 


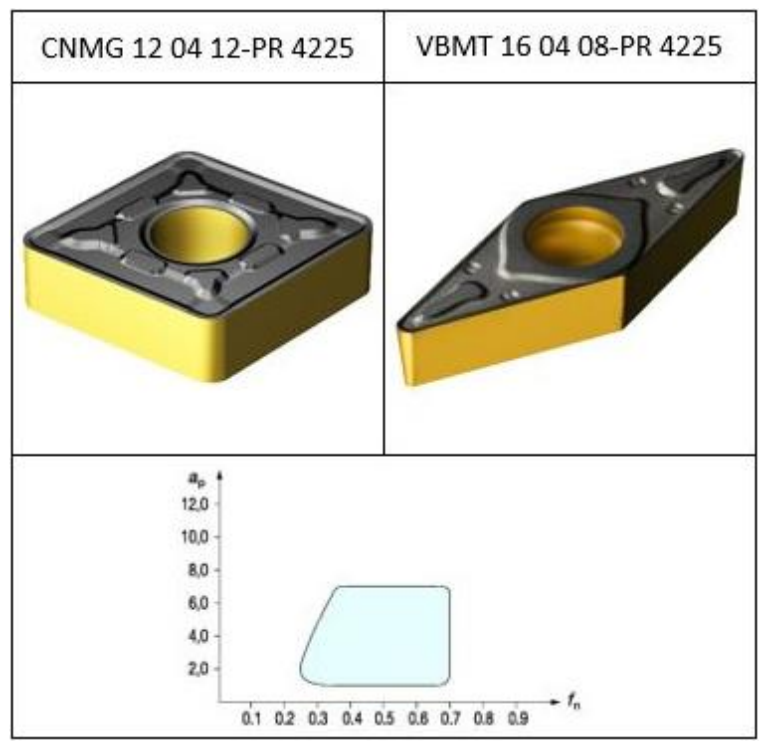

Fig. 4: Used turning inserts. a) Left side: roughing insert, b) Right side: Finishing insert and c) Bottom: recommended cutting conditions.

Tab. 3: Cutting parameters of turning operations.

\begin{tabular}{cccc}
\hline Parameter & Roughing & Semi finishing & Finishing \\
\hline $\begin{array}{c}\text { Cutting speed } \\
\mathrm{V}_{\mathrm{c}}(\mathrm{m} / \mathrm{min}) \\
\quad \text { Feed } \\
\mathrm{f}(\mathrm{mm} / \mathrm{rev})\end{array}$ & 190 & 300 & 300 \\
$\begin{array}{c}\text { Cutting depth } \\
\quad \begin{array}{l}\text { a }(\mathrm{mm}) \\
\text { Number of steps }\end{array}\end{array}$ & 0.4 & 0.2 & 0.2 \\
\end{tabular}

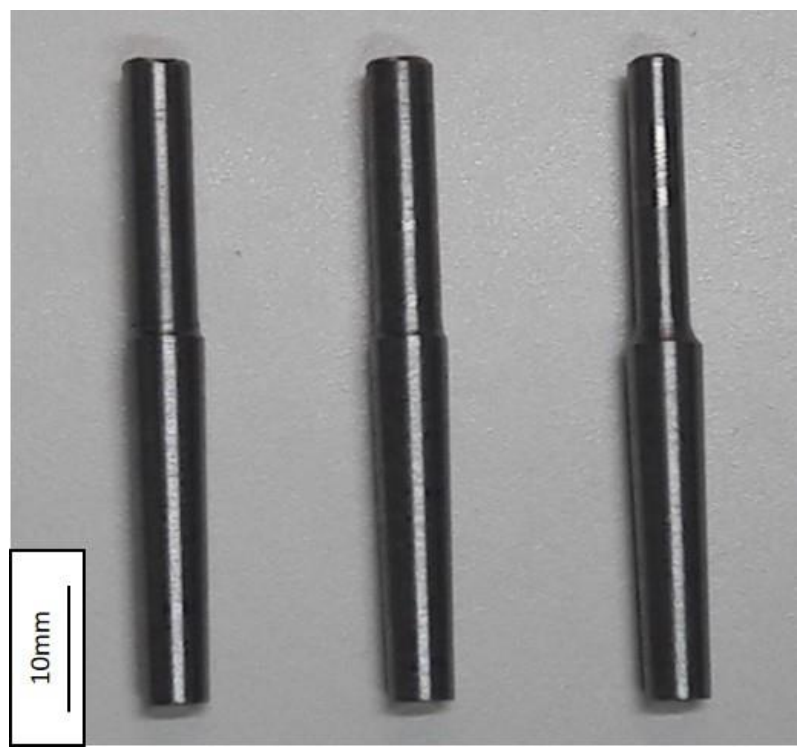

Fig. 5: FSAM tool core geometry.

\subsection{Electrodeposition operation}

Firstly, an electroplated tool is a tool provided with various abrasive grains on a surface of a parent material by electroplating. The last step in the manufacturing process of this tool is the electroplating, which consists of the addition of abrasive grains to the core of the tool.

In this case, the denomination of the grains used are SK$16-20-R 1,2$, and the grains were electroplated in a single layer.

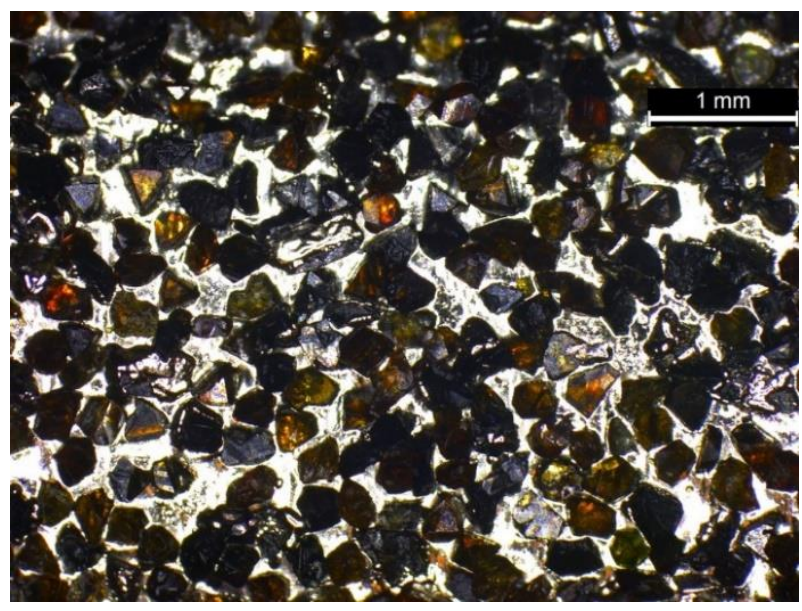

Fig. 6: Dimensions of abrasive grain size measured with microscope.

When carrying out the electrodeposition of abrasive grains, the grains distribution is randomly performed along the entire surface (see the distribution in Fig.6). Nevertheless, the distribution of abrasive grains in electroplated tools has a direct influence on the lifetime of the tool and processing efficiency.

In this way a series of advantages are achieved.

- Better removal rates and less thermal damage to the part.

- Hold simple and intricate forms for their entire life of the tool.

- Lower long-term cost with the potential reduction or elimination of time associated with set-up, dressing and tool changes. 
- Reusable tools after a flaking process, followed by a re-electroplating operation. The proccess is shown in Fig. 7.

\section{Worn tool}

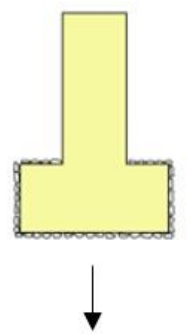

\section{Flaking process}

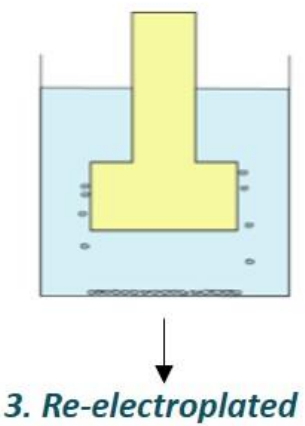

tool

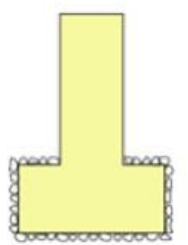

Fig. 7: Diagram of re-electroplating operation.

\section{CONCLUSIONS}

This work was mainly focused on the manufacturing process of a SAM tool to be used in the semi-finishing and finishing operations of a blade. Firstly, the tool geometry was designed according to a developed mathematic algorithm that, given a concrete geometry to be manufactured as an input, offers as the output the geometry of a tool that best fits the surface in a flank milling operation.

Major conclusions from this work can be summarized, concerning the manufacturing process of a SAM tool, as given below

- Electroplated tools are widely used for grinding because the abrasive grains. So, it is reasonable to think about the use of these tools in the semifinishing or finishing operations of complex geometries like blisks or impellers, which require very good surface quality.

- Electroplated diamond tools are able to retain their original shape and dimensions during their entire working life. This reason makes the SAM process a possible alternative to the conventional milling process, where the tool wear could be uncontrolled with a cutting edge breakage. Hence, it is likely to use these types of tools in order to obtain geometries that need to be strictly precise.
- Just about any shape can be plated with almost any size of diamond grain. Electroplating can also provide production cost savings because the part's finish shape can be easily produced by the plated tool.

- Other important point in the manufacturing of SAM tools is the quantity of abrasive grains, which influences the tool lifetime and processing efficiency. If the abrasive grain electroplating amount is too large, clogging and welding occurred, and the surface quality of manufactured part decreased.

- Electroplating allows diamond particles to protrude from the bond matrix, providing a faster cutting action with minimum heat generation.

Therefore, the use of SAM tools together with flank milling strategies combines the benefits from milling and grinding technologies using conventional multitasking machining centres.

\section{REFERENCES}

[Chaves-Jacob 2012] Chaves-Jacob, J., Poulachon, G. and Duc, E. Optimal strategy for finishing impeller blades using 5-axis machining. International Journal of Advanced Manufacturing Technology. January 2012, Vol.58, No.5-8., pp 573583. ISSN 0268-3768

[Klocke 2015] Klocke, F. et al. Technological and Economical Assessment of Alternative Process Chains for Blisk Manufacture. Procedia CIRP. 2015, Vol.35, pp 6772. ISSN 2212-8271

[Wang 2018] Wang, G. et al. Multi-parameter optimization of machining impeller surface based on the on-machine measuring technique. Chinese Journal of Aeronautics. October 2018. ISSN 1000-9361

[Menzel 2004] Menzel, C., Bedi, S. and Mann, S. Triple tangent flank milling of ruled surfaces. Computer-Aided Design. March 2004, Vol.36, No.3, pp 289296. ISSN 00104485

[Zhu 2010] Zhu, L. et al. Global optimization of tool path for five-axis flank milling with a conical cutter. Computer-Aided Design. October 2010, Vol.42, No.10, pp 903910. ISSN 0010-4485

[Yi 2018] Yi, J. et al. Optimized tool path planning for fiveaxis flank milling of ruled surfaces using geometric decomposition strategy and multi-population harmony search algorithm. Applied Soft Computing. December 2018, Vol.73, pp 547561. ISSN 1568-4946

[Li 2019] Li, Z.L. and Zhu, L.M. Compensation of deformation errors in five-axis flank milling of thin-walled parts via tool path optimization. Precision Engineering. January 2019, Vol.55, pp 7787. ISSN 0141-6359

[Chuang 2007] Chuang, L.C. and Young, H.T. Integrated rough machining methodology for centrifugal impeller manufacturing. International Journal of Advanced Manufacturing Technology. November 2007, Vol.34, No.11-12., pp 10621071. ISSN 0268-3768

[Gong 2005] Gong, H., Cao, L.X and Jian, L. Improved positioning of cylindrical cutter for flank milling ruled surfaces. Computer-Aided Design. October 2005, Vol.37, No.12., pp 12051213. ISSN 0010-4485

[Lu 2016] Lu, Y.A., Bi, Q.Z. and Zhu, L.M. Five-axis flank milling of impellers: Optimal geometry of a conical tool considering stiffness and geometric constraints. Proceedings of the Institution of Mechanical Engineers, 
Part B: Journal of Engineering Manufacture. January 2016, Vol.230, No.1., pp 3852

[Bo 2016] Bo, P. et al. Towards efficient 5-axis flank CNC machining of free-form surfaces via fitting envelopes of surfaces of revolution. Computer-Aided Design. October 2016, Vol.79, pp 111. ISSN 0010-4485

[Petrilli 2012] Petrilli, R. Super abrasive machining for PM. Metal Powder Report. December 2012, Vol.67, No.6., pp 3841. ISSN 0026-0657

[Stephenson 2006] Stephenson, D.J., Sun, X. and Zervos, C. A study on ELID ultra precision grinding of optical glass with acoustic emission. International Journal of Machine Tools and Manufacture. August 2006, Vol.46, No.10., pp 10531063. ISSN 0890-6955

[Biermann 2017] Biermann, D., Bathe, T. and Rautert, C. Core Drilling of Fiber Reinforced Materials using Abrasive Tools. Procedia CIRP. 2017, Vol.66, pp 175180. ISSN 2212-8271

[González 2018] González, H. et al. Super Abrasive Machining of Integral Rotary Components Using Grinding Flank Tools. MDPI-Metals. January 2018, Vol.8, No.1., pp 24. ISSN 2075-4701
[Lin 1998] Lin, C.R. and Kuo, C.T. Improvement of mechanical properties of electroplated diamond tools by microwave plasma CVD diamond process. Surface and Coatings Technology. November 1998, Vol.110, No.1-2., pp 1923. ISSN 0257-8972

[De Resende 2001] De Resende, L.W. et al. Multi-layer structure for chemical vapor deposition diamond on electroplated diamond tools. Diamond and Related Materials. March-July 2001, Vol.10, No.3-7., pp332336. ISSN 0925-9635

[Tanaka 1997] Tanaka, T. and Isono, Y. Influences of metal constituents to the characteristics and grinding abilities of metal bonded diamond wheel. Journal of Materials Processing Technology. January 1997, Vol.63, No.1-3., pp175180. ISSN 0924-0136

[Tsai 2012] Tsai, M.Y. and Jian, S.X. Development of a micro-graphite impregnated grinding wheel. International Journal of Machine Tools and Manufacture. May 2012, Vol.56, No.1-3., pp94101. ISSN 0890-6955 